\title{
Coping strategies mediate the associations between COVID-19 experiences and mental health outcomes in pregnancy
}

\author{
Jennifer E. Khoury ${ }^{1}$ (1) $\cdot$ Leslie Atkinson ${ }^{2} \cdot$ Teresa Bennett $^{3,4} \cdot$ Susan M. Jack ${ }^{4,5} \cdot$ Andrea Gonzalez $^{3,4}$
}

Received: 23 January 2021 / Accepted: 13 April 2021 / Published online: 19 June 2021

(c) The Author(s), under exclusive licence to Springer-Verlag GmbH Austria, part of Springer Nature 2021

\begin{abstract}
The COVID-19 pandemic has resulted in elevated mental health problems for pregnant women. Effective coping strategies likely reduce the impact of COVID-19 on mental health. This study aimed to (1) understand how COVID-19 stressors are related to different coping strategies and (2) identify whether coping strategies act as mechanisms accounting for the associations between COVID-19 stressful experiences and mental health problems in pregnancy. Participants were 304 pregnant women from Ontario, Canada. Depression, anxiety, insomnia, and perceived stress were assessed using validated measures. COVID-related stressors (i.e., financial difficulties, social isolation), subjective negative impact of COVID-19, and coping strategies used in response to COVID-19 were assessed by questionnaires. Results indicated that the subjective negative impact of COVID-19 was associated with more dysfunctional coping and less emotion-focused coping, whereas specific COVID-19 stressors, namely financial difficulties and social isolation, were associated with more dysfunctional coping and problem-focused coping. Dysfunctional coping was linked to elevated mental health problems and emotion-focused coping was linked to less mental health problems. Dysfunctional coping and emotion-focused coping partially mediated the effects of specific COVID-19 stressors on mental health outcomes. Findings indicate that coping is one pathway through which the COVID-19 pandemic impacts mental health in pregnancy. Supports and interventions for pregnant women during the pandemic should focus on bolstering coping skills, in order to minimize the mental health consequences of COVID-19.
\end{abstract}

Keywords Pregnancy $\cdot$ Coronavirus $\cdot$ COVID- $19 \cdot$ Mental health $\cdot$ Coping

\section{Introduction}

The COVID-19 pandemic is causing elevated distress across populations. Systematic reviews and meta-analyses, integrating research from across the globe, indicate that a substantial

Women's mental health during the Covid-19 pandemic Edited by: Anita Riecher-Rössler

Jennifer E. Khoury

Jennifer.khoury@msvu.ca

1 Department of Psychology, Mount Saint Vincent University, Halifax, NS, Canada

2 Department of Psychology, Ryerson University, Toronto, ON, Canada

3 Department of Psychiatry and Behavioural Neurosciences, McMaster University, Hamilton, ON, Canada

4 Offord Centre for Child Studies, McMaster University, Hamilton, ON, Canada

5 School of Nursing, McMaster University, Hamilton, ON, Canada portion of the general population is experiencing high rates of depression (25-34\%) and anxiety (25-32\%) during this pandemic (Bueno-Notivol et al. 2021; Ren et al. 2020; Salari et al. 2020). Similarly, pregnant and perinatal women are experiencing comparably elevated rates of depression (25-31\%), anxiety (34-42\%), and psychological distress (70\%) (Fan et al. 2020; Sun et al. 2020; Yan et al. 2020). Pre-COVID research shows that pregnancy is a period of vulnerability, characterized by higher prevalence of anxiety and depression compared to the general population (meta-analyses: Dennis et al. 2017; McCance-Katz 2019; Woody et al. 2017). While mental health difficulties appear similarly elevated in pregnant and nonpregnant samples during the COVID-19 pandemic, prenatal mental health problems increase risk for adverse birth effects and developmental outcomes (meta-analyses: Grigoriadis et al. 2018; Madigan et al. 2018). Importantly, the degree to which COVID-19 impacts mental health is variable. Effective coping strategies likely act as a protective factor that mitigates the impact of COVID-19 on mental health. Indeed, engaging in self-care, including physical activity and healthy eating, is 
linked to better wellbeing during the pandemic (Davenport et al. 2020; Deng et al. 2020; Farewell et al. 2020; Lebel et al. 2020). However, it remains unclear how specific coping strategies impact mental health outcomes in pregnant women during COVID-19.

Coping is a primary component of an individual's response to stressful events (Folkman and Lazarus 1980). Research has distinguished between three major types of coping: (1) problem-focused coping, which involves actions aimed at addressing the problem (e.g., planning, seeking instrumental support), (2) emotion-focused coping, which aims to manage negative emotions (e.g., seeking emotional support, cognitive restructuring), and (3) dysfunctional coping, which involves maladaptive strategies that are not helpful in dealing with the stressor (e.g., denial, behavioral disengagement) (Carver 1997; Folkman and Moskowitz 2004). Indeed, dysfunctional coping has been associated with adverse mental health outcomes in pregnant women across different circumstances (Chen et al. 2020; Guardino and Dunkel Schetter 2014; GutiérrezZotes et al. 2015; Lau et al. 2016). In contrast, the effects of problem-focused and emotion-focused coping are mixed, though these strategies tend to be associated with more positive outcomes in pregnant women (Guardino and Dunkel Schetter 2014 for review). Furthermore, different forms of coping have been shown to act as mechanisms/mediators between stressful experiences and mental health outcomes in pregnancy (Choi et al. 2015; Greene et al. 2021; Giurgescu et al. 2006; Zhang et al. 2020).

Several studies have shown that effective coping strategies are associated with better psychological wellbeing during the COVID-19 pandemic, including reduced anxiety and depression (e.g., Brehl et al. 2021; Groarke et al. 2020; Jiang et al. 2020; Jungmann and Witthöft 2020; Fullana et al. 2020). In addition, two studies have specifically examined the role of coping as a mediator between stress and mental health outcomes during the COVID-19 pandemic. In a sample of college students, Ye et al. (2020) showed that COVID-19 stress was related to more maladaptive coping and less adaptive coping strategies, and that adaptive coping mediated the link between COVID-19 stress and acute stress disorder. In contrast, in a sample of the general public, maladaptive coping mediated the effect of intolerance of uncertainty on mental health outcomes (Rettie and Daniels 2020). Additional research is needed to determine which forms of coping act as mediators linking COVID-19 stress and mental health in pregnant women.

\section{The current study}

Identifying the pathways through which COVID-19 is associated with mental health in pregnancy is necessary to inform public health initiatives aimed at ameliorating the mental health outcomes of this pandemic. The present study examined how the subjective impact of COVID-19 and COVID-19 stressors (financial, social isolation) were associated with coping and mental health outcomes in pregnant women. The objectives of this study were to (1) determine how COVID-19 stressful experiences are associated with different forms of coping; (2) examine how coping strategies are related to mental health, and (3) identify whether coping strategies act as mediators of the associations between COVID-19 experiences and mental health problems in pregnancy.

\section{Methods}

\section{Participants}

Participants were 304 pregnant women between 19 and 44 years old ( $\mathrm{M}=32.09, \mathrm{SD}=4.27$ years) and 4 to 36 weeks gestation ( $M=21.44$ weeks, $\mathrm{SD}=8.93$ weeks) (see Table 1 for sample characteristics). Participants completed online surveys between June and July 2020. This was a convenience sample; recruitment was primarily conducted through social media advertisements (eliciting participant in a study assessing how COVID-19 impacts wellbeing during pregnancy). Inclusion criteria were that individuals (1) live in Ontario, Canada, (2) read and write in English, (3) be 18 years of age or older, and (4) be $\leq 36$ weeks gestation. This study was approved by the Hamilton Integrated Research Ethics Board under Project \#11,034.

\section{Measures}

Centre for Epidemiologic Studies Depression Scale The 10-item Centre for Epidemiologic Studies Depression Scale (CESD) (Andresen et al. 1994) captured the presence of depressive symptoms over the past 7 days. Responses range from 0 "rarely or never (less than 1 day)" to 3 "most or all of the time (5-7 days)". The CES-D total score ranges from 0 to 30, a cutoff score of $\geq 10$ indicates the presence of clinically significant depressive symptoms (Andresen et al. 1994).

Generalized Anxiety Disorder-7 The 7-item Generalized Anxiety Disorder-7 (GAD-7) screens for GAD symptoms occurring in the past 2 weeks (Spitzer et al. 2006), with responses ranging from 0 "not at all" to 3 "nearly every day". The GAD-7 total score ranges from 0 to 21 , a cutoff score of $\geq 10$ indicates possible clinical levels of anxiety.

Cambridge Worry Scale The 16-item Cambridge Worry Scale (CWS) (Statham et al. 1997) was used to assesses pregnancyspecific anxiety and general anxiety. Participants indicated the degree to which they worry about each item, on a scale of 0 ("not a worry") to 5 ("major worry").

Insomnia Severity Index The 7-item Insomnia Severity Index (ISI) (Bastien et al. 2001) measures sleep satisfaction, sleep interference, 
Table 1 Sample characteristics

\begin{tabular}{|c|c|}
\hline & $\begin{array}{l}\text { Sample } \\
\text { characteristics } \\
\%(N)\end{array}$ \\
\hline \multicolumn{2}{|l|}{ Gestation } \\
\hline First trimester & $24.3 \%(74)$ \\
\hline Second trimester & $45.1 \%(137)$ \\
\hline Third trimester & $30.6 \%(93)$ \\
\hline \multicolumn{2}{|l|}{ Number of children } \\
\hline 0 & $47.4 \%(144)$ \\
\hline 1 & $35.5 \%(108)$ \\
\hline 2 & $13.2 \%(40)$ \\
\hline$\geq 3$ & $4.0 \%(12)$ \\
\hline \multicolumn{2}{|l|}{ Marital status } \\
\hline Married & $80.9 \%(246)$ \\
\hline Common law & $13.5 \%(41)$ \\
\hline In a relationship, but not married or common law & $3.3 \%(10)$ \\
\hline Divorced & $0.7 \%(2)$ \\
\hline Separated & $0.3 \%(1)$ \\
\hline Single & $1.3 \%(4)$ \\
\hline \multicolumn{2}{|l|}{ Ethnicity } \\
\hline White & $84.9 \%(258)$ \\
\hline Asian & $6.9 \%(21)$ \\
\hline Indigenous & $0.7 \%(2)$ \\
\hline Multiple ethnicities & $3.0 \%(9)$ \\
\hline Other & $4.6 \%(14)$ \\
\hline \multicolumn{2}{|l|}{ Education } \\
\hline Less than high school & $0.3 \%(1)$ \\
\hline High school & $3.6 \%(11)$ \\
\hline Non-university postsecondary & $24.8 \%(75)$ \\
\hline Bachelor's degree & $38.6 \%(117)$ \\
\hline Above bachelor's degree & $32.7 \%(99)$ \\
\hline \multicolumn{2}{|l|}{ Annual family income } \\
\hline$<\$ 20,000$ & $1.0 \%(3)$ \\
\hline$\$ 20,000$ to $\$ 34,999$ & $5.7 \%(17)$ \\
\hline$\$ 35,000$ to $\$ 69,999$ & $11.1 \%(33)$ \\
\hline$\$ 70,000$ to $\$ 89,999$ & $13.5 \%(40)$ \\
\hline$\$ 90,000$ to $\$ 109,999$ & $15.8 \%(47)$ \\
\hline$\$ 110,000$ to $\$ 149,999$ & $29.6 \%(88)$ \\
\hline$\$ 150,000$ to $\$ 199,999$ & $15.5 \%(46)$ \\
\hline$\geq 200,000$ & $7.7 \%(23)$ \\
\hline
\end{tabular}

worry about sleep, and the severity of insomnia problems. The ISI total score ranges from 0 to 28 ; scores between 0 and 7 indicate no clinically significant insomnia, scores between 8 and 14 indicate subthreshold insomnia, and scores between 15 and 28 indicate clinical insomnia (moderate to severe severity).

Perceived Stress Scale The 10-item Perceived Stress Scale (PSS) assesses experiences of stress in the past month (Cohen and Williamson 1988), with responses ranging from 1 "never" to 4 "very often." The PSS total score ranges from 0 to 40 ; scores between 0 and 13 indicate low stress, scores between 14 and 26 indicate moderate stress, and scores between 27 and 40 indicate high perceived stress (Cohen and Williamson 1988).

COVID-19 stressful experiences Participants reported how they have been impacted by the COVID-19 pandemic (see specific items in Table 2). A single item captured the subjective overall impact of COVID-19; responses ranged from 1 (Very Positive) to 5 (Very Negative). In addition, six items ranging from 1 (Not at $A l l$ ) to 7 (A lot) were used to assess the financial difficulties and four dichotomous items $(0=$ No, $1=$ Yes $)$ captured social isolation resulting from COVID-19. Composites were created by averaging the financial difficulties items ${ }^{1}$ and summing the isolation items. Given the small number of items on each scale, internal consistency was assessed using average inter-item correlations (Briggs and Cheek 1986; Herman 2015) (Table 2).

Brief Coping Strategies The 28-item Brief Coping Strategies (Brief COPE) (Carver 1997) measured coping strategies used in response to the stress of COVID-19. Participants rated their use of each coping strategy on a scale ranging from 1 "I haven't been doing this at all" to 4 "I've being doing this a lot." In accordance with prior work (e.g., Chen et al. 2020; Cooper et al. 2008), three Brief COPE subscales were created: dysfunctional coping (Denial, Venting, Self-Distraction, Substance Use, Behavioral Disengagement, and Self-Blame), problem-focused coping (Active Coping, Planning, and Using Instrumental Support), and emotion-focused coping (Using Emotional Support, Positive Reframing, Acceptance, Humor, and Religion).

\section{Statistical analyses}

Descriptive and correlation analyses were conducted using SPSS 26. Confirmatory factor analysis (CFA) was used to create a latent variable from the five mental health and distress questionnaire total scores. Structural equation mediation models (SEM) were conducted in Mplus Version 8 using full information maximum likelihood (FIML) and bootstrapping (with bias-corrected confidence intervals) to account for missing data (Fox 2015; Shrout and Bolger 2002). Three SEM models tested the direct paths between the specific COVID-19 stressors (financial, isolation, subjective impact of COVID-19) on the mental health, and the indirect paths through (1) dysfunctional coping, (2) emotionfocused coping, and (3) problem-focused coping.

Covariates that had significant bivariate correlations with any mental health measure were entered in the final step of

\footnotetext{
${ }_{1}$ Two financial difficulties items pertained to partner's salary and job and thus were not applicable for women who were not in a relationship. The financial difficulties composite average was based only on applicable items.
} 
Table 2 Descriptive statistics

\begin{tabular}{|c|c|c|c|}
\hline Measure & $\begin{array}{l}\mathrm{M}(\mathrm{SD}) / \\
\%(\mathrm{~N})\end{array}$ & Range & Internal consistency \\
\hline \multicolumn{4}{|l|}{ COVID-19 experiences } \\
\hline Financial composite & & & $r_{\text {inter-item }}=.44$ \\
\hline I lost income due to COVID-19 & $2.83(2.34)$ & $1-7$ & \\
\hline My partner lost income due to COVID-19 & $2.40(1.98)$ & $1-7$ & \\
\hline My job security is vulnerable because of COVID-19 & $2.54(2.05)$ & $1-7$ & \\
\hline My partner's job security is vulnerable because of COVID-19 & $2.55(1.92)$ & $1-7$ & \\
\hline I have difficulty paying my rent or mortgage because of COVID-19 & $1.76(1.34)$ & $1-7$ & \\
\hline Loss of income affects the kind of groceries I buy & $2.29(1.84)$ & $1-7$ & \\
\hline Isolation composite & & & $r_{\text {inter-item }}=.22$ \\
\hline I don't have as much contact with friends because of COVID-19 & $95.7 \%(291)$ & $0-1$ & \\
\hline I don't have as much contact with a close family member because of COVID-19 & $87.2 \%(265)$ & $0-1$ & \\
\hline I am not going to my place of work because of COVID-19 & $70.7 \%(215)$ & $0-1$ & \\
\hline $\begin{array}{l}\text { I have been under self-quarantine (which means that I have only had contact with my } \\
\text { immediate household members) because of COVID-19 }\end{array}$ & $42.4 \%(129)$ & $0-1$ & \\
\hline \multicolumn{4}{|l|}{ Subjective impact of COVID } \\
\hline $\begin{array}{l}\text { Taking everything about COVID-19 into account, the effects of COVID-19 on me } \\
\text { and my household have been }\end{array}$ & $3.71(0.72)$ & $2-5$ & \\
\hline \multicolumn{4}{|l|}{ Mental health and distress } \\
\hline Center for Epidemiologic Studies Depression Scale (CES-D) & $\begin{array}{l}11.49(6.35) \\
57.1 \%(173)\end{array}$ & $0-26$ & $\alpha=.87$ \\
\hline Generalized Anxiety Disorder-7 (GAD-7) & $\begin{array}{l}7.27(5.07) \\
30.0 \%(91)\end{array}$ & $0-21$ & $\alpha=.90$ \\
\hline Insomnia Severity Index (ISI) & $\begin{array}{l}9.67(5.67) \\
19.2 \%(58)\end{array}$ & $0-28$ & $\alpha=.88$ \\
\hline Perceived Stress Scale (PSS) & $\begin{array}{l}19.71(7.02) \\
18.5 \%(56)\end{array}$ & $1-38$ & $\alpha=.90$ \\
\hline Cambridge Worry Scale (CWS) & $30.09(15.30)$ & $0-71$ & $\alpha=.88$ \\
\hline \multicolumn{4}{|l|}{ Coping } \\
\hline Brief dysfunctional coping strategies & $20.76(4.47)$ & $10-36$ & $\alpha=.70$ \\
\hline Brief problem-focused coping strategies & $14.21(3.72)$ & $4-24$ & $\alpha=.76$ \\
\hline Brief emotion-focused coping strategies & $24.35(5.20)$ & $11-38$ & $\alpha=.76$ \\
\hline
\end{tabular}

Note: Means and SD are provided for continuous items, \% and (N) are provided to indicate positive endorsement of dichotomous items. The \% and $(\mathrm{N})$ of the sample over the cut score is provided, for clinical depression (CES-D), clinical anxiety (GAD), high stress (PSS), and clinical sleep difficulties (ISI). Financial difficulties item responses range from 1 (Not at All) to 7 (A lot). Isolation composite item responses range from 0 (No) to 1 (Yes). Subjective impact of COVID-19 ranged from 1 (Very Positive) to 5 (Very Negative). $r_{\text {inter-item }}$ represent average inter-item correlations for scale items. Optimal values range between 0.2 and 0.4 (Briggs and Cheek 1986)

the modelling; covariates that significantly $(p<0.05)$ contributed to the model were retained. Model fit was assessed using the comparative fit index (CFI), Tucker-Lewis index (TLI), root mean squared error of approximation (RMSEA), and standardized root mean square residual (SRMR). Values equal to or greater than 0.95 for CFI and TLI and equal to or less than 0.08 for RMSEA and SRMR are considered indicative of good model fit (Schreiber et al. 2006).

Missing data $0.3 \%(k=1)$ of the CES-D, GAD, PSS, and Brief COPE, $1.0 \%(k=3)$ of the ISI, and $2.0 \%(k=6)$ of the CWS. None of the COVID-19 items was missing.

\section{Results}

\section{Descriptive statistics}

Table 2 displays the descriptive statistics and psychometric properties for COVID-19 experiences, mental health, and coping measures.

COVID-19 stressful experiences The continuous items related to financial difficulties were averaged to compute a financial composite $(\mathrm{M}=2.40, \mathrm{SD}=1.38)$ and the 
dichotomous items related to social isolation were summed to derive the isolation composite $(\mathrm{M}=2.96, \mathrm{SD}=0.97)$. Regarding subjective appraisal of the impact of COVID$19,4.9 \%$ of the sample indicated that the overall effect of COVID-19 has been Very Negative effect, $72.0 \%$ indicated a Negative effect, $12.2 \%$ indicated No effect, $10.9 \%$ indicated a Positive effect, and zero participants indicated a Very Positive effect of COVID-19.

Mental health and distress Descriptively, $57.1 \%$ of the sample scored $\geq 10$ on the CES-D and $30.0 \%$ scored $\geq 10$ on the GAD-7, indicating clinically significant levels of depression and anxiety, respectively. In total, $18.8 \%$ of the sample reported low levels of stress, $62.7 \%$ reported moderate stress, and $18.5 \%$ reported high levels of stress. Lastly, $39.5 \%$ of the sample reported no clinically significant insomnia, $41.2 \%$ reported subthreshold insomnia, and $19.2 \%$ reported clinical insomnia (see Table 2).

\section{Bivariate correlations}

As shown in Table 3, education, income, ethnicity, and number of children were significantly correlated with different mental health outcomes, and were tested as covariates in subsequent analyses.

\section{Confirmatory factor analysis}

As shown in Table 4, the CFA measurement model had good model fit and all indicators loaded significantly onto the mental health latent variable. This latent variable was used as the outcome variable in subsequent SEM mediation analyses.

\section{Structural equation mediation analyses}

Subjective impact of COVID-19, coping, and mental health The SEM model examining the indirect paths from the subjective impact of COVID-19 to mental health via coping (dysfunctional, problem-focused, emotion-focused) demonstrated good model fit (Fig. 1). The final model included significant $(p<0.05)$ covariates (ethnicity and education). As shown in Fig. 1, the subjective negative impact of COVID-19 was significantly associated with greater dysfunctional coping and less emotion-focused coping, but was not associated with problem-focused coping ( $a$ paths). Dysfunctional coping was positively associated with greater mental health severity and emotion-focused coping was negatively related to mental health ( $b$ paths). In addition, the association between the subject impact of COVID-19 and mental health remained significant after taking coping strategies into account (direct effect). Finally, the indirect effects through dysfunctional coping and emotion-focused coping were significant; the indirect path through problem-focused coping was not significant. This indicates that the subjective impact of COVID-19 is indirectly linked to increased mental health symptoms, through elevated dysfunctional coping and reduced emotion-focused coping.

COVID-19 financial difficulties, coping, and mental health The SEM model examining the indirect paths from COVID-19 financial difficulties to mental health via coping is shown in Fig. 2. Greater financial difficulties were associated with more dysfunctional coping and problem-focused coping, but not emotion-focused coping. Only dysfunctional coping and emotion-focused coping were associated with mental health severity. Although there was not a significant direct association between COVID financial difficulties and mental health severity (Fig. 2), indirect effects can occur in the absence of a direct effect (Hayes 2017). Only the indirect effect through dysfunctional coping was significant (Fig. 2).

COVID-19 social isolation, coping, and mental health Similarly, more social isolation was significantly associated with dysfunctional coping and problem-focused coping, and dysfunctional coping and emotion-focused coping were associated with mental health severity. Finally, COVID-related isolation was indirectly linked to increased mental health severity, through elevated dysfunctional coping, but not other forms of coping (Fig. 3).

\section{Discussion}

To our knowledge, this is the first study to examine how coping strategies mediate associations between COVID-19 stressors and mental health problems during pregnancy. The findings demonstrate that (1) the perceived negative impact of COVID-19 as well as financial difficulties and social isolation resulting from COVID-19 are associated with distinct coping strategies; (2) coping strategies are linked to mental health; and (3) dysfunctional and emotion-focused coping strategies mediate the impact of COVID-19 experiences on mental health problems in pregnancy. Taken together, these findings indicate that coping is one pathway through which the pandemic is impacting mental health in pregnancy, highlighting a viable target for interventions aimed at minimizing the mental health consequences of COVID-19.

The present findings are in line with the burgeoning literature, including several meta-analyses (e.g., Fan et al. 2020; Hessami et al. 2020; Sun et al. 2020; Yan et al. 2020), which indicate that pregnant women are experiencing significant mental health problems during COVID-19. Specifically, $57 \%$ of the sample endorsed clinically elevated depression, 30\% reported clinically elevated anxiety, 19\% 


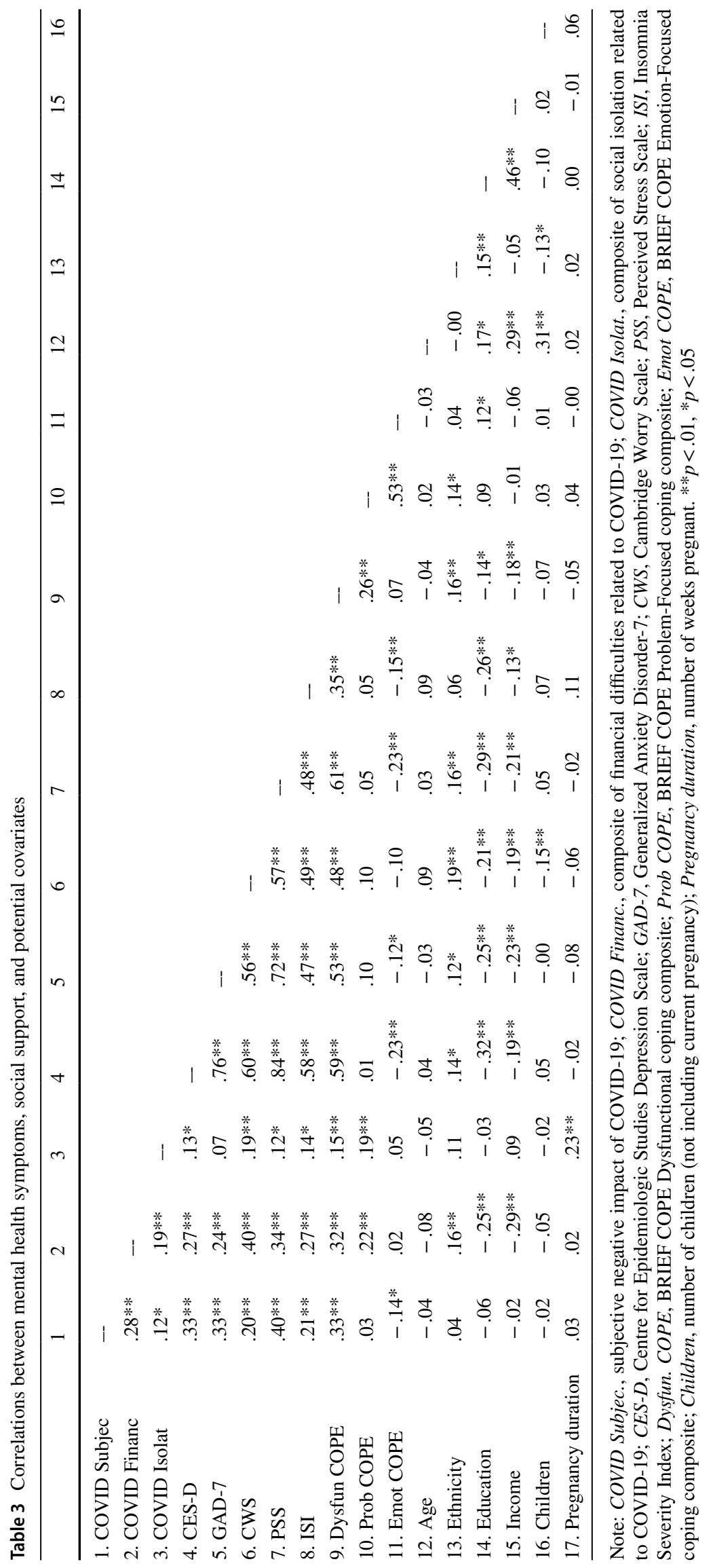


Table 4 Confirmatory factor analysis to derive mental health and distress latent composite

\begin{tabular}{lllllll}
\hline Mental health/distress & $\begin{array}{l}\text { Unstandardized } \\
\text { estimate }\end{array}$ & $\begin{array}{l}\text { Standardized } \\
\text { estimate }\end{array}$ & SE & Est/SE & 95\% CI & $p$ \\
\hline Depression (CES-D) & 1.00 & 0.94 & 0.01 & 75.49 & {$[0.92,0.97]$} & 0.00 \\
Generalized anxiety (GAD-7) & 0.68 & 0.81 & 0.02 & 34.44 & {$[0.76,0.85]$} & 0.00 \\
Worry (CWS) & 1.68 & 0.65 & 0.04 & 18.08 & {$[0.58,0.72]$} & 0.00 \\
Perceived Stress (PSS) & 1.03 & 0.88 & 0.02 & 54.93 & {$[0.85,0.91]$} & 0.00 \\
Sleep difficulties (ISI) & 0.57 & 0.60 & 0.04 & 15.11 & {$[0.52,0.67]$} & 0.00 \\
\hline
\end{tabular}

Note: Model fit: $\mathrm{CFI}=0.99 ; \mathrm{TLI}=.97$; RMSEA $=.09 ; \mathrm{SRMR}=.03 . C E S-D$, Centre for Epidemiologic Studies Depression Scale; GAD-7, Generalized Anxiety Disorder-7; CWS, Cambridge Worry Scale; PSS, Perceived Stress Scale; ISI, Insomnia Severity Index. SE standard error; Est/SE, estimate/SE; 95\% CI, 95\% confidence interval; SE, Est/SE, and $95 \% \mathrm{CI}$ are provided based on the standardized estimate

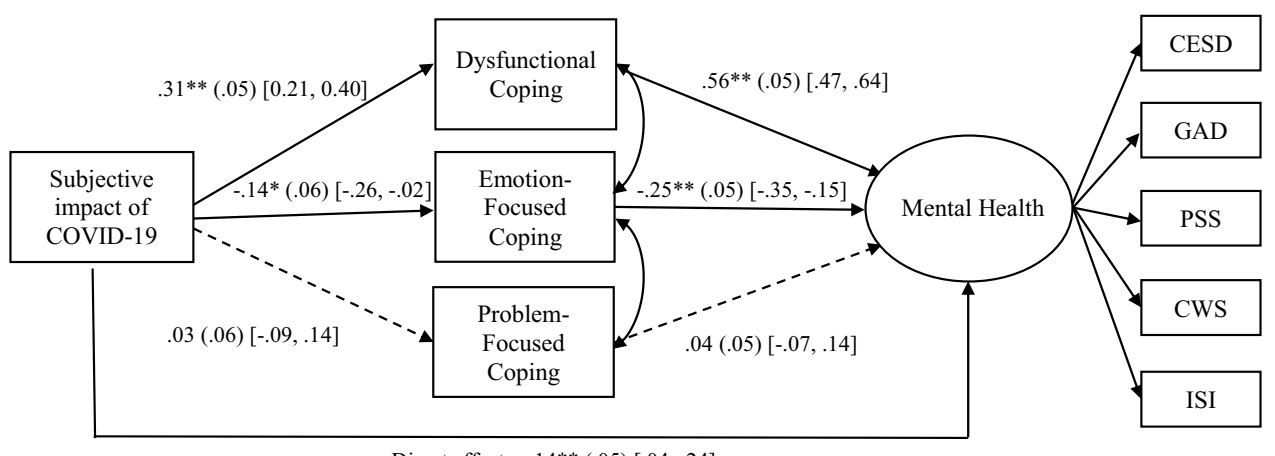

Direct effect $=.14^{* *}(.05)[.04, .24]$

Fig. 1 Structural Equation Model: The subjective impact of COVID19 is indirectly linked to mental health through dysfunctional and emotion-focused coping strategies. Note: $\mathrm{CFI}=.98 ; \mathrm{TLI}=.97$; RMSEA $=.05 ;$ SRMR $=.03$. Standardized beta coefficients, standard error, and 95\% confidence intervals are shown. Model includes ethnicity and education as covariates. The indirect paths through dysfunctional coping $(\beta=.17 * *, \mathrm{SE}=.03,95 \%$ CI $[.11, .24])$ and emotion-focused coping $(\beta=.04 *, \mathrm{SE}=.02,95 \% \mathrm{CI}[.01, .07])$ were significant; the indirect path through problem-focused coping was not significant $(\beta=.001, \mathrm{SE}=.004,95 \% \mathrm{CI}[-.01, .01])$. The total effect was significant $\left(\beta=.35^{*}, \mathrm{SE}=.05,95 \%\right.$ CI $\left.[.25, .45]\right)$. CES-D $=$ Centre for Epidemiologic Studies Depression Scale, GAD-7=Generalized Anxiety Disorder-7, CWS = Cambridge Worry Scale, PSS $=$ Perceived Stress Scale, ISI $=$ Insomnia Severity Index. $* p<.05, * * p<.01$

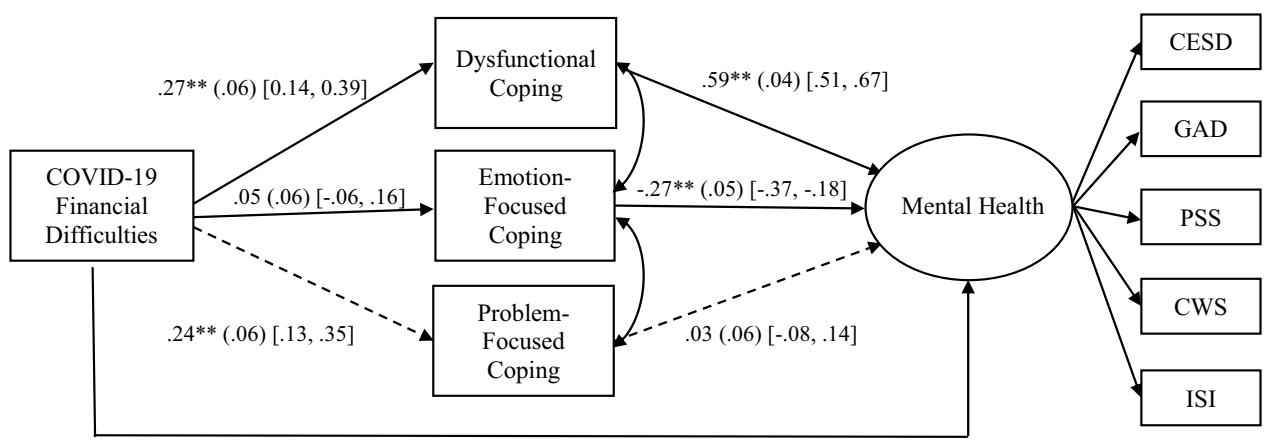

Direct effect $=.07(.05)[-.03, .16]$

Fig. 2 Structural Equation Model: COVID-19 financial difficulties are indirectly linked to mental health through dysfunctional coping strategies. Note: $\mathrm{CFI}=.97 ; \mathrm{TLI}=.94 ; \mathrm{RMSEA}=.07 ; \mathrm{SRMR}=.03$. Standardized beta coefficients, standard error, and 95\% confidence intervals are shown. Model includes ethnicity and education as covariates. The indirect paths through dysfunctional coping was significant $\left(\beta=.16^{* *}, \mathrm{SE}=.04,95 \% \mathrm{CI}[.08, .24]\right)$; the indirect paths through emotion-focused coping $(\beta=-.01, \mathrm{SE}=.02,95 \% \mathrm{CI}[-.04$, $.02])$ and problem-focused coping ( $\beta=.01, \mathrm{SE}=.01,95 \% \mathrm{CI}[-.02$, .04]) were not significant. The total effect was significant $(\beta=.22 * *$, $\mathrm{SE}=.06,95 \%$ CI $[.10, .34])$. CES-D = Centre for Epidemiologic Studies Depression Scale, GAD-7 $=$ Generalized Anxiety Disorder-7, CWS $=$ Cambridge Worry Scale, PSS $=$ Perceived Stress Scale, $\mathrm{ISI}=$ Insomnia Severity Index. ${ }^{*} p<.05, * * p<.01$ 


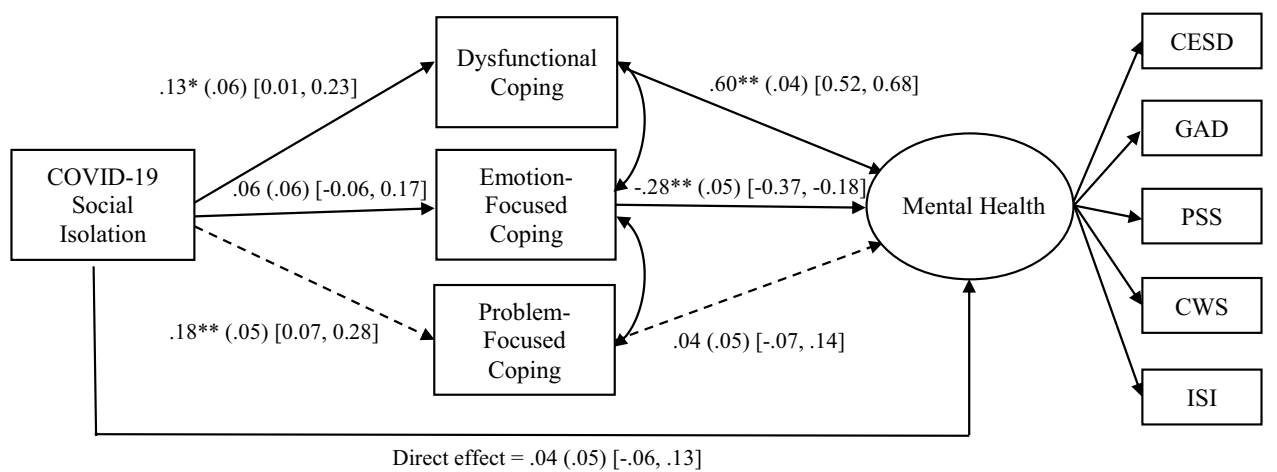

Fig. 3 Structural Equation Model: COVID-19 social isolation is indirectly linked to mental health through dysfunctional coping strategies. Note: $\mathrm{CFI}=.98 ; \mathrm{TLI}=.97 ; \mathrm{RMSEA}=.05 ; \mathrm{SRMR}=.03$. Standardized beta coefficients, standard error, and $95 \%$ confidence intervals are shown. Model includes ethnicity and education as covariates. The indirect paths through dysfunctional coping was significant $\left(\beta=.08^{*}\right.$, $\mathrm{SE}=.03,95 \% \mathrm{CI}[.01, .14])$; the indirect paths through emotion-

reported elevated insomnia, and $81 \%$ reported moderate to severe levels of stress. In comparison, in Ontario, Canada, prior to COVID-19, 7.7\% of pregnant women experienced clinically elevated depression and $9.1 \%$ experienced anxiety (Public Health Ontario 2017). These findings are also in line with nation-wide studies and longitudinal studies showing greater severity of mental health problems experienced during COVID-19 compared to prior to the pandemic (Berthelot et al. 2020; Moyer et al. 2020; López-Morales et al. 2021; Parra-Saavedra et al. 2020). This work, in combination with prior research, underscores the need to address the heightened mental health problems experienced during the pandemic.

In addition, the present findings underscore the importance of coping in the context of COVID-19. We found that particular COVID-19-related experiences were differentially associated with distinct forms of coping. Specifically, individuals who appraised the COVID-19 pandemic to have a greater negative impact engaged in more dysfunctional coping and less emotion-focused coping. In contrast, greater financial difficulties and social isolation were associated with more dysfunctional coping and problem-focused coping, but not emotion-focused coping. Negative cognitive appraisal represents a global index of the psychological impact of COVID-19, which might be more associated with coping strategies that target the emotional outcomes of COVID-19, as opposed to problem-focused coping which targets specific problems related to COVID19. For example, someone who is feeling overwhelmed by the overall impact of COVID-19 might seek out emotional support from others, whereas someone who is experiencing financial strain resulting from the pandemic might plan ways of obtaining instrumental support (e.g., governmental assistance). focused coping $(\beta=-.02, \mathrm{SE}=.02,95 \% \mathrm{CI}[-.05, .02])$ and problem-focused coping $(\beta=.01, \mathrm{SE}=.01,95 \% \mathrm{CI}[-.01, .03])$ were not significant. The total effect was not significant $(\beta=.10, \mathrm{SE}=.06,95 \%$ CI $[-.01, .22])$. CES-D $=$ Centre for Epidemiologic Studies Depression Scale, GAD-7 = Generalized Anxiety Disorder-7, CWS $=$ Cambridge Worry Scale, PSS $=$ Perceived Stress Scale, ISI=Insomnia Severity Index. ${ }^{*} p<.05, * * p<.01$

In line with prior research in pre-COVID pregnancy samples (e.g., Guardino and Dunkel Schetter 2014; Lau et al. 2016), we found that dysfunctional coping was related to elevated mental health problems, emotion-focused coping was associated with less mental health problems, whereas problem-focused coping was not related to mental health. These findings add to the larger literature showing that specific coping strategies are associated with better/worse mental health outcomes during the COVID-19 pandemic (Jiang et al. 2020; Jungmann and Witthöft 2020). For example, in a non-pregnant sample, global use of maladaptive coping strategies (i.e., withdrawal, blame, self-devaluation), measured before the pandemic, was associated with elevated stress and anxiety during the pandemic, whereas adaptive coping strategies were not associated with mental health problems (Brehl et al. 2021). Prior work hypothesized that emotion-focused coping is more effective in the context of uncontrollable stressors, whereas problem-focused coping is more effective in response to controllable situations (e.g., Folkman and Moskowitz 2004). Thus, it is possible that in the current study only emotion-focused coping was related to reduced mental health problems because of the uncontrollable nature of the COVID-19 pandemic.

Finally, the present findings indicate that dysfunctional and emotion-focused coping strategies act as mediators through which COVID-19 experiences are indirectly associated with mental health in pregnancy. These findings are in line with prior research with pregnant women, indicating that coping mediates associations between various stressful experiences and mental health problems (Choi et al. 2015; Greene et al. 2021; Giurgescu et al. 2006; Zhang et al. 2020). Interestingly, some research shows that coping can also moderate the impact of stress on mental health (Chen et al. 2020; Lau et al. 2014). The present findings indicate 
that distinct coping strategies can be targeted to mitigate the effects of different COVID-19 experiences on mental health in pregnancy. Specific intervention targets can be prioritized depending on one's perceptions of the impact of COIVD-19 and the particular COVID-19-related stressors experienced. First, reducing the degree of dysfunctional coping (i.e., denial, disengagement, self-blame) may reduce the burden on mental health. Second, for individuals who perceive the global impact of COVID-19 to be more negative, engaging in more emotion-focused coping (e.g., seeking emotional support, positively reframing) can also reduce the impact on mental health. Taken together, promoting the use of adaptive coping strategies in place of dysfunctional coping strategies can help to ameliorate the effect of COVID-19 on mental health.

The COVID-19 pandemic likely affects the coping strategies that individuals are able to use. For example, ways of relieving stress and seeking emotional support, such as physical activity, attending counselling or support groups, and face-to-face contact with loved ones, have been altered during the pandemic. Indeed, a portion of the present sample was unable to access message therapy and counselling services, although the majority continued to have prenatal services (albeit remotely) (see Khoury et al. 2021). Related research shows that self-care activities, including exercise and mediation (Davenport et al. 2020; Deng et al. 2020), as well as social support and access to support groups (Dib et al. 2020; Khoury et al. 2021) are associated with better mental health outcomes for pregnant women during COVID-19. Finding new ways of effectively coping during the pandemic is essential to positive mental health outcomes.

In addition, we found that greater mental health and distress symptoms were experienced by those who identified as non-White and who reported lower education and less income. This is in line with research indicating that ethnicminority status and financial stress predict elevated mental health problems in pregnant women during COVID-19 (Deng et al. 2020; Preis et al. 2021; Thayer and Gildner 2020). Ethnic minorities and low-income individuals might experience greater stress as a result of COVID-19 (e.g., greater financial concerns) and have less access to particular coping strategies (e.g., internet access to have face-to-face virtual interactions). Therefore, public health initiatives must not only take these sociodemographic factors into account when identifying those at greatest risk of mental health outcomes but also ensure that a health equity-oriented lens is applied to any policy or clinical responses.

The present findings can be used to inform public health and mental health initiatives aimed at limiting the mental health consequences of COVID-19. Professionals working with pregnant women should inquire about different stressful experiences (financial strain, feelings of isolation, etc.), as well as the ways in which individuals typically cope with these stressors. Health care professionals can provide psychoeducation regarding the effectiveness of different coping strategies. Furthermore, an important aspect of intervention will be for individuals to learn when to apply different coping strategies based on the specific situational demands, as some situations might require an emotion-focused approach, whereas others require a problem-focused approach.

The limitations of this study should be considered. First, this is a cross-sectional study wherein we used a regression-based statistical approach to model mediation effects, which precludes any inference of causality. Although all measures were completed at a single time point, the coping measure was worded so that participants indicated how they were coping in response to the COVID-19 pandemic. However, it is possible that individuals' coping styles impact how they attribute COVID-19 stressors. Future longitudinal research is needed to ascertain temporal precedence between COVID-19 stressors and coping and mental health outcomes. Second, a convenience sample of participants was requited through social media and online questionnaires were used in this study. Future work is needed to determine whether results can be replicated with a more representative sample of the general population, using varied forms of assessments. Third, given the rapid onset of COVID-19, this is the first use of the COVID-19 Experience Scale in the published literature. Although we show acceptable internal consistency and validity, future research is needed to further establish the psychometric properties of this scale. Lastly, this is a socio-demographically low-risk sample, in that most participants were well educated and had higher income. This sample was also low risk in terms of health concerns related to COVID-19 and few participants were diagnosed with COVID-19. Future work is needed to determine whether similar coping strategies are used in response to COVID-19 stressors in more socio-demographically diverse samples who may be coping with greater health burden.

\section{Conclusions}

This study augments prior reports that pregnant women are experiencing elevated mental health problems in response to the COVID-19 pandemic. The present findings indicate that coping strategies are directly linked to mental health outcomes and that dysfunctional coping and emotion-focused coping mediate the associations between COVID-19 experiences and mental health outcomes in pregnancy. These findings highlight the need for widely available interventions to bolster adaptive coping skills in order to limit the mental health burden of pregnant women during the COVID-19 pandemic. 
Acknowledgements We would like to thank all of the pregnant people who participated in this research as well as the research assistants who worked on this project.

Author contribution All authors contributed to the study conception and design. Material preparation was performed by Jennifer E. Khoury, Leslie Atkinson, and Andrea Gonzalez. Data collection and analysis were performed by Jennifer E. Khoury. The first draft of the manuscript was written by Jennifer E. Khoury and all authors commented on previous versions of the manuscript. All authors read and approved the final manuscript.

Funding This work was supported by a Tier II Canadian Institute of Health Research (CIHR) Canadian Research Chair (CRC) in Family Health and Preventive Interventions (Project\#: 20011917) awarded to Dr. Andrea Gonzalez and by a CIHR Postdoctoral Fellowship awarded to Dr. Jennifer Khoury.

\section{Declarations}

Ethics approval This study was approved by the Hamilton Integrated Research Ethics Board under Project \#11,034, on June 3, 2020.

Conflict of interest The authors declare no competing interests.

\section{References}

Andresen EM, Malmgren JA, Carter WB, Patrick DL (1994) Screening for depression in well older adults: evaluation of a short form of the CES-D. Am J Prev Med 10(2):77-84

Bastien CH, Vallières A, Morin CM (2001) Validation of the Insomnia Severity Index as an outcome measure for insomnia research. Sleep Med 2(4):297-307

Berthelot N, Lemieux R, Garon-Bissonnette J, Drouin-Maziade C, Martel É, Maziade M (2020) Uptrend in distress and psychiatric symptomatology in pregnant women during the coronavirus disease 2019 pandemic. Acta Obstet Gynecol Scand 99(7):848-855

Brehl A-K, Schene A, Kohn N, Fernández G (2021) Maladaptive emotion regulation strategies in a vulnerable population predict increased anxiety during the Covid-19 pandemic: A pseudo-prospective study. J Affect Disord 4:100113

Briggs SR, Cheek JM (1986) The role of factor analysis in the development and evaluation of personality scales. J Pers 54(1):106-148

Bueno-Notivol J, Gracia-García P, Olaya B, Lasheras I, López-Antón R, Santabárbara J (2021) Prevalence of depression during the COVID-19 outbreak: a meta-analysis of community-based studies. Int J Clin Health Psychol 21(1):100196

Carver CS (1997) You want to measure coping but your protocol's too long: consider the brief cope. Int J Behav Med 4(1):92-100

Chen T, Laplante DP, Elgbeili G, Brunet A, Simcock G, Kildea S, King S (2020) Coping during pregnancy following exposure to a natural disaster: the QF2011 Queensland Flood Study. J Affect Disord 273:341-349

Choi KW, Sikkema KJ, Velloza J, Marais A, Jose C, Stein DJ, ..., Joska JA (2015) Maladaptive coping mediates the influence of childhood trauma on depression and PTSD among pregnant women in South Africa. Arch Women's Mental Health 18(5): 731-738

Cohen S, Williamson G (1988) Perceived Stress Scale [Instrument]. Published instrument
Cooper C, Katona C, Livingston G (2008) Validity and reliability of the brief COPE in carers of people with dementia: the LASERAD Study. J Nerv Ment Dis 196(11):838-843

Davenport MH, Meyer S, Meah VL, Strynadka MC, Khurana R (2020) Moms are not OK: COVID-19 and maternal mental health. Frontiers in Global Women's Health. https://doi.org/10. 3389/fgwh.2020.00001

Deng J, Hu C, Ning Y, Xu P, Lu C, Wang, J, ..., Duan P (2020) Physical exercise and economic burden associated with anxiety symptoms in pregnant women during the COVID-19 pandemic. https://doi.org/10.21203/rs.3.rs-105483/v1

Dennis CL, Falah-Hassani K, Shiri R (2017) Prevalence of antenatal and postnatal anxiety: systematic review and meta-analysis. $\mathrm{Br}$ J Psychiatry 210(5):315-323

Dib S, Rougeaux E, Vázquez-Vázquez A, Wells JC, Fewtrell M (2020) Maternal mental health and coping during the COVID19 lockdown in the UK: data from the COVID-19 New Mum Study. Int J Gynecol Obstet 151(3):407-414

Fan S, Guan J, Cao L, Wang M, Zhao H, Chen L, Yan L (2020) Psychological effects caused by COVID-19 pandemic on pregnant women: a systematic review with meta-analysis. Asian J Psychiatry: 102533

Farewell CV, Jewell J, Walls J, Leiferman JA (2020) A mixed-methods pilot study of perinatal risk and resilience during COVID19. J Prim Care Community Health 11:2150132720944074

Folkman S, Lazarus RS (1980) An analysis of coping in a middleaged community sample. J Health Soc Behav 21(4):219-239

Folkman S, Moskowitz JT (2004) Coping: pitfalls and promise. Annu Rev 55:745-774

Fox J (2015) Applied regression analysis and generalized linear models. Sage Publications, Los Angeles

Fullana MA, Hidalgo-Mazzei D, Vieta E, Radua J (2020) Coping behaviors associated with decreased anxiety and depressive symptoms during the COVID-19 pandemic and lockdown. J Affect Disord 275:80-81

Giurgescu C, Penckofer S, Maurer MC, Bryant FB (2006) Impact of uncertainty, social support, and prenatal coping on the psychological well-being of high-risk pregnant women. Nurs Res 55(5):356-365

Greene CA, McCoach DB, Briggs-Gowan MJ, Grasso DJ (2021) Associations among childhood threat and deprivation experiences, emotion dysregulation, and mental health in pregnant women. Psychol Trauma. https://doi.org/10.1037/tra0001013

Grigoriadis S, Graves L, Peer M, Mamisashvili L, Tomlinson G, Vigod SN, ..., Cheung A (2018) Maternal anxiety during pregnancy and the association with adverse perinatal outcomes: systematic review and meta-analysis. J Clin Psychiatry 79(5): 17r12011

Groarke JM, Berry E, Graham-Wisener L, McKenna-Plumley PE, McGlinchey E, Armour C (2020) Loneliness in the UK during the COVID-19 pandemic: cross-sectional results from the COVID-19 Psychological Wellbeing Study. PLoS One 15(9):e0239698

Guardino CM, Dunkel Schetter C (2014) Coping during pregnancy: a systematic review and recommendations. Health Psychol Rev 8(1):70-94

Gutiérrez-Zotes A, Labad J, Martín-Santos R, García-Esteve L, Gelabert E, Jover M, ..., Gratacós M (2015) Coping strategies and postpartum depressive symptoms: a structural equation modelling approach. Eur Psychiatry 30(6): 701-708

Hayes AF (2017) Introduction to mediation, moderation, and conditional process analysis: a regression-based approach. Guilford Publications

Herman BC (2015) The influence of global warming science views and sociocultural factors on willingness to mitigate global warming. Sci Educ 99(1):1-38 
Hessami K, Romanelli C, Chiurazzi M, Cozzolino M (2020) COVID19 pandemic and maternal mental health: a systematic review and meta-analysis. J Matern Fetal Neonatal Med: 1-8

Jiang HJ, Nan J, Lv ZY, Yang J (2020) Psychological impacts of the COVID-19 epidemic on Chinese people: exposure, post-traumatic stress symptom, and emotion regulation. Asian Pac J Trop Med 13(6):252-259

Jungmann SM, Witthöft M (2020) Health anxiety, cyberchondria, and coping in the current COVID-19 pandemic: which factors are related to coronavirus anxiety? J Anxiety Disord 73:102239

Khoury JE, Atkinson L, Bennett T, Jack SM, Gonzalez A (2021) COVID-19 and mental health during pregnancy: the importance of cognitive appraisal and social support. J Affect Disord 282:1161-1169

Lau Y, Wang Y, Ho Keung Kwong D, Wang Y (2014) Testing direct and moderating effects of coping styles on the relationship between perceived stress and antenatal anxiety symptoms. J Psychosom Obstet Gynecol 36(1):29-35

Lau Y, Wang Y, Kwong DH, Wang Y (2016) Are different coping styles mitigating perceived stress associated with depressive symptoms among pregnant women? Perspect Psychiatr Care 52(2):102-112

Lebel C, MacKinnon A, Bagshawe M, Tomfohr-Madsen L, Giesbrecht G (2020) Elevated depression and anxiety symptoms among pregnant individuals during the COVID-19 pandemic. J Affect Disord 277(1):5-13

López-Morales H, Del Valle MV, Canet-Juric L, Andrés ML, Galli JI, Poó F, Urquijo S (2021) Mental health of pregnant women during the COVID-19 pandemic: a longitudinal study. Psychiatry Res 295:113567

Madigan S, Oatley H, Racine N, Fearon RP, Schumacher L, Akbari E, ..., Tarabulsy GM (2018) A meta-analysis of maternal prenatal depression and anxiety on child socioemotional development. J Am Acad Child Adolesc Psychiatry 57(9): 645-657

McCance-Katz EF (2019) The national survey on drug use and health: 2017. Substance Abuse and Mental Health Services Administration. https://www.samhsa.gov/data/sites/default/files/nsduh-ppt09-2018.pdf. Accessed May, 7

Moyer CA, Compton SD, Kaselitz E, Muzik M (2020) Pregnancyrelated anxiety during COVID-19: a nationwide survey of 2740 pregnant women. Arch Women's Mental Health: 1-9

Ontario Agency for Health Protection and Promotion (Public Health Ontario) (2017) Snapshots: data file for maternal health snapshot (2013 to 2015) [Internet]. Toronto, ON: Queen's Printer for Ontario; [cited 2018 May 14]. Available from: http://www.publi chealthontario.ca/en/DataAndAnalytics/Snapshots/Pages/Mater nal-health.aspx. Accessed 20 Dec 2020

Parra-Saavedra M, Villa-Villa I, Pérez-Olivo J, Guzman-Polania L, Galvis-Centurion P, Cumplido-Romero Á, Santacruz-Vargas D, Rivera-Moreno E, Molina-Giraldo S, Guillen-Burgos H, Navarro E, Flórez-Lozano K, Barrero-Ortega A, Sanz-Cortes M, Miranda J (2020) Attitudes and collateral psychological effects of COVID-19 in pregnant women in Colombia. Int J Gynecol Obstet 151(2):203-208
Preis H, Mahaffey B, Pati S, Heiselman C, Lobel M (2021) Adverse perinatal outcomes predicted by prenatal maternal stress among US women at the COVID-19 pandemic onset. Ann Behav Med 55(3):179-191

Ren X, Huang W, Pan H, Huang T, Wang X, Ma Y (2020) Mental health during the Covid-19 outbreak in China: a meta-analysis. Psychiatr Q: 1-13

Rettie H, Daniels J (2020) Coping and tolerance of uncertainty: predictors and mediators of mental health during the COVID-19 pandemic. Am Psychol. Advance online publication. https://doi.org/ 10.1037/amp0000710

Salari N, Hosseinian-Far A, Jalali R, Vaisi-Raygani A, Rasoulpoor S, Mohammadi M, ..., Khaledi-Paveh B (2020) Prevalence of stress, anxiety, depression among the general population during the COVID-19 pandemic: a systematic review and meta-analysis. Global Health 16(1): 1-11

Schreiber JB, Nora A, Stage FK, Barlow EA, King J (2006) Reporting structural equation modeling and confirmatory factor analysis results: a review. J Educ Res 99(6):323-338

Shrout PE, Bolger N (2002) Mediation in experimental and nonexperimental studies: new procedures and recommendations. Psychol Methods 7(4):422

Spitzer RL, Kroenke K, Williams JB, Löwe B (2006) A brief measure for assessing generalized anxiety disorder: the GAD-7. Arch Intern Med 166(10):1092-1097

Statham H, Green JM, Kafetsios K (1997) Who worries that something might be wrong with the baby? A prospective study of 1072 pregnant women. Birth 24(4):223-233

Sun F, Zhu J, Tao H, Ma Y, Jin W (2020) A systematic review involving 11,187 participants evaluating the impact of COVID-19 on anxiety and depression in pregnant women. J Psychosom Obstet Gynecol: 1-9

Thayer ZM, Gildner TE (2020) COVID-19-related financial stress associated with higher likelihood of depression among pregnant women living in the United States. Am J Hum Biol: e23508

Woody CA, Ferrari AJ, Siskind DJ, Whiteford HA, Harris MG (2017) A systematic review and meta-regression of the prevalence and incidence of perinatal depression. J Affect Disord 219:86-92

Yan H, Ding Y, Guo W (2020) Mental health of pregnant and postpartum women during the coronavirus disease 2019 pandemic: a systematic review and meta-analysis. Front Psychol 11:3324

Ye Z, Yang X, Zeng C, Wang Y, Shen Z, Li X, Lin D (2020) Resilience, social support, and coping as mediators between COVID-19related stressful experiences and acute stress disorder among college students in China. Appl Psychol Health Well Being. https:// doi.org/10.1111/aphw.12211

Zhang X, Li JH, Wang J, Li J, Long ZT, Cao FL (2020) Childhood neglect and psychological distress among pregnant women: the chain multiple mediation effect of perceived social support and positive coping. J Nerv Ment Dis 208(10):764-770

Publisher's note Springer Nature remains neutral with regard to jurisdictional claims in published maps and institutional affiliations. 